\title{
Correction to: Induced membrane maintains its osteogenic properties even when the second stage of Masquelet's technique is performed later
}

Florelle Gindraux $^{1,2} \cdot$ François Loisel $^{1,2} \cdot$ Michael Bourgeois $^{1} \cdot$ Karim Oudina $^{3} \cdot$ Martine Melin $^{4} \cdot$ Benoit de Billy $^{2,5}$. Pauline Sergent ${ }^{1}$ - Gregoire Leclerc ${ }^{1}$ - Hervé Petite ${ }^{3} \cdot$ Frederic Auber $^{2,5} \cdot$ Laurent Obert $^{1,2} \cdot$ Isabelle Pluvy $^{1,2}$

Published online: 30 October 2019

(c) Springer-Verlag GmbH Germany, part of Springer Nature 2019

\section{Correction to: \\ European Journal of Trauma and Emergency Surgery https://doi.org/10.1007/s00068-019-01184-4}

The original version of this article unfortunately contained a mistake. The presentation and legends of Figs. 4 and 5 were incorrect. The corrected versions are given below. The original article has been corrected.

The original article can be found online at https://doi.org/10.1007/ s00068-019-01184-4.

Florelle Gindraux

fgindraux@chu-besancon.fr

1 Orthopaedic and Traumatology Surgery Department,

University Hospital of Besancon, Besancon, France

2 Nanomedicine Lab, Imagery and Therapeutics (EA 4662), SFR FED 4234, University of Franche-Comté, Besancon, France

3 Laboratory of Bioengineering and Biomechanics for Bone Articulation (B2OA-UMR CNRS 7052), University Paris Diderot, Paris, France

4 Novotec, Bron, France

5 Paediatric Surgery Department, University Hospital of Besancon, Besancon, France 


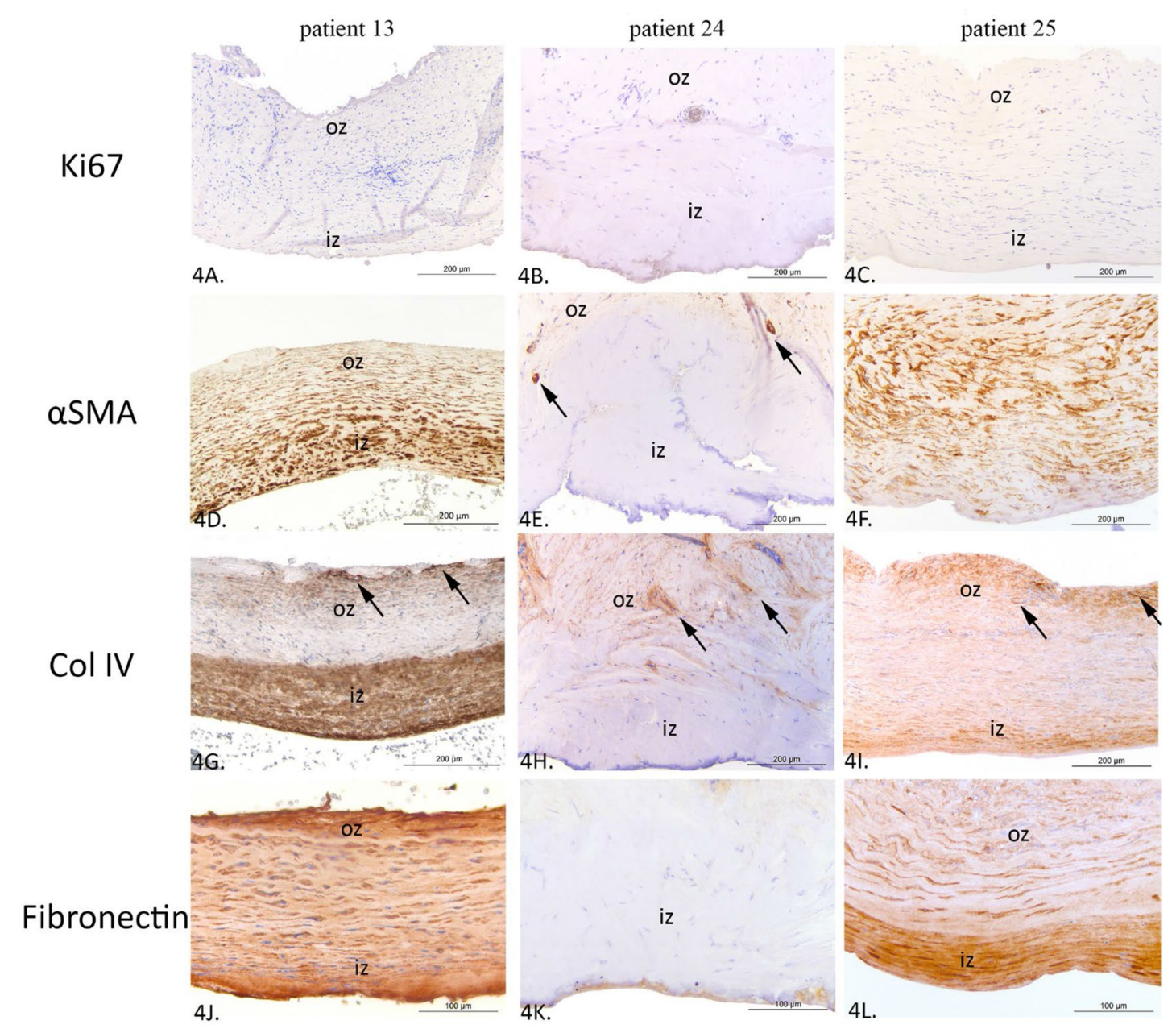

Fig. 4 Immunolabelling of IMs sampled at the time of the second procedure: 4 months for patient (P) 13 (4a, d, g, j), 13.5 months for P24 (4b, e, h, k) and 14.7 months for P25 (4c, f, i, l): anti-human $\mathrm{Ki67}$ (4a, b, c), anti-human alpha-smooth muscle actin ( $\alpha \mathrm{SMA})$ (4d, e, f), anti-human collagen IV (Col IV) (4g, h, i) and anti-human fibronectin $(4 \mathrm{j}, \mathrm{k}, \mathrm{l})$. Blood vessels are indicated by arrows (diagonal upward arrow). Outermost zone (oz) and innermost zone (iz) are visible 


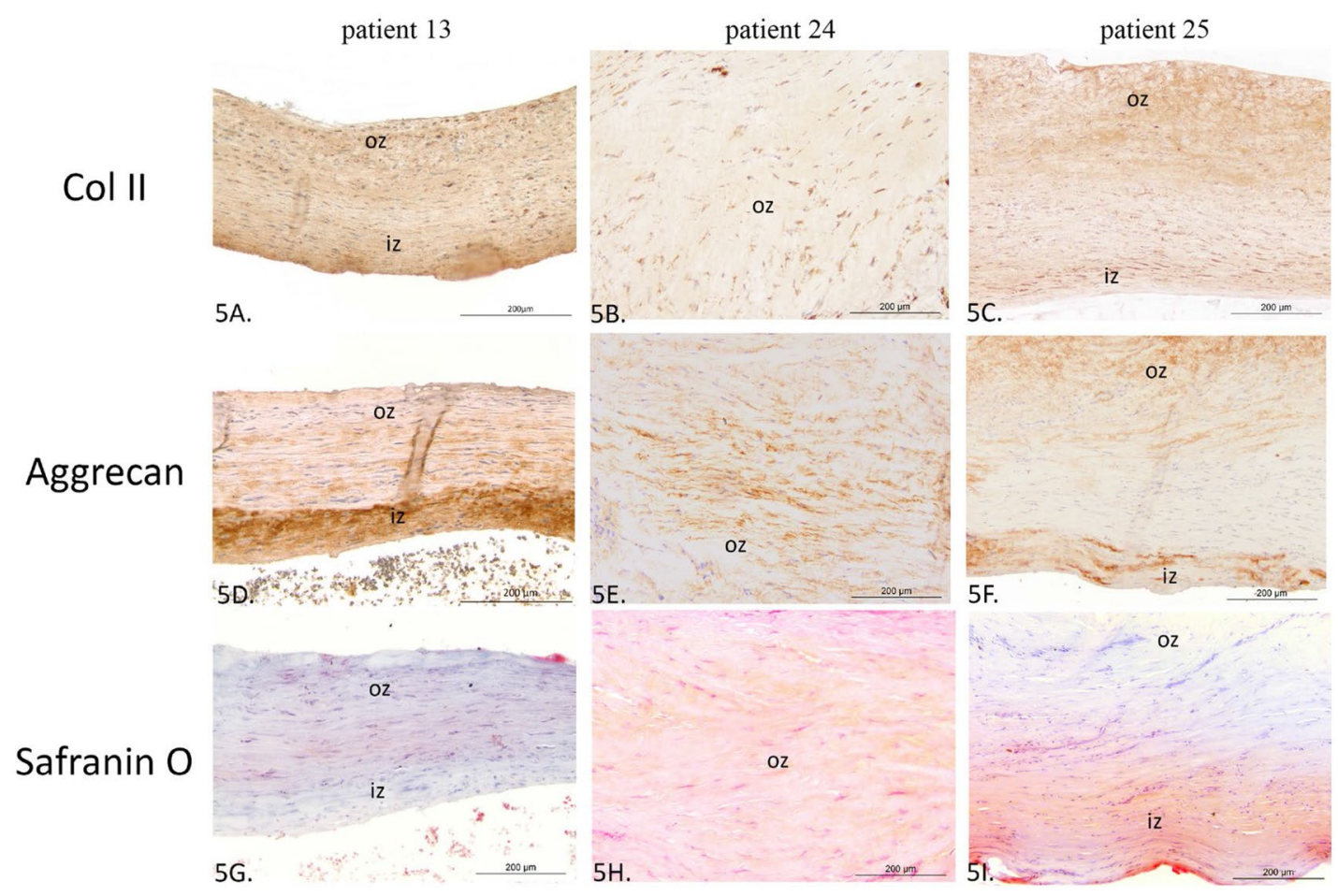

Fig. 5 Immunolabelling specific to cartilage in IMs sampled at the time of the second procedure: 4 months for patient $(\mathrm{P}) 13(5 \mathrm{a}, \mathrm{d}, \mathrm{g})$, 13.5 months for P24 (5b, e, h) and 14.7 months for P25 (5c, f, i): anti-

human collagen II (Col II) (5a, b, c) and anti-human aggrecan (5d, e, f), and Safranin O staining (5g, h, i). Outermost zone (oz) and innermost zone (iz) are visible 